\title{
Glycogen Storage Disease Type I Presenting with Hypertension During Infancy
}

\author{
Eshita Bhowmik • Moumita Ghosh • Tapas Kumar Sabui • \\ Rakesh Mondal
}

Received: 7 October 2014 / Accepted: 19 February 2015 / Published online: 5 March 2015

(C) Dr. K C Chaudhuri Foundation 2015

To the Editor: Glycogen storage disease (GSD) type 1 is an autosomal recessive disorder characterized by absence or deficiency of glucose 6- phosphatase in liver, kidney and intestinal mucosa. Renal disease and hypertension are rare complications of GSD type 1 in childhood. A 6-mo-old boy presented with fever, cough and respiratory distress. After clinical and laboratory evaluation a provisional diagnosis of cardiac failure due to hypertension in a case of GSD type 1 with infection (probably viral) was made. The child was put on mechanical ventilator, treated with labetalol infusion, furosemide, antibiotics and glucose bolus. After reduction of the blood pressure and improvement of cardiac failure, oral antihypertensives namely, labetalol, amlodipine, enalapril and furosemide were used to bring the blood pressure below 95th percentile. He was put on frequent daytime and nighttime feed with soy based milk formula, and prohibited from eating foods containing galactose, fructose and sucrose. His liver biopsy showed glycogen and fat vacuoles. USG revealed mildly enlarged kidneys. Echocardiography was normal. Kidney biopsy showed focal mesengial proliferation and basement membrane thickening. Follow up visits after 6 mo showed improving levels of serum lipids and uric acid and blood pressure below 90th percentile. We plan to add allopurinol and fibrate if required and taper off furosemide. Renal disease is a late complication in GSD type 1 occuring after $20 \mathrm{y}$ of age in cases who have been ineffectively treated [1]. First manifestation of renal disease is glomerular hyperfiltration, which may occur in younger patients [1,2]. Later microalbuminemia, proteinuria, hypertension, hematuria, nephrocalcinosis may occur, finally leading to end stage renal disease. Earliest pathological finding is focal segmental glomerulosclerosis $[1,3]$. Hypertension is hypothesized to be due to activation of angiotensin system [4]. There is only one case report of hypertension and proteinuria observed in a 2-y-old child with type IA GSD [5]. Dietary therapy improves metabolic abnormalities and risk of long term complications [1].

Conflict of Interest None.

Source of Funding None.

\section{References}

1. Chen YT. Type I, glycogen storage disease: kidney involvement, pathogenesis and its treatment. Pediatr Nephrol. 1991;5:71-6.

2. Baker L, Dahlem S, Goldfarb S, et al. Hyperfiltration and renal disease in glycogen storage disease, type I. Kidney Int. 1989;35:1345-50.

3. Chen YT, Coleman RA, Scheinman JI, Kolbeck PC, Sidbury JB. Renal disease in type I glycogen storage disease. N Engl J Med. 1988;318:7-11.

4. Yiu WH, Pan CJ, Ruef RA, et al. Angiotensin mediates renal fibrosis in the nephropathy of glycogen storage disease type Ia. Kidney Int. 2008;73:716-23.

5. Jonas AJ, Verani RR, Howell RR, Conley SB. Hypertension in a child with type IA glycogen storage disease. Am J Kidney Dis. 1988;11: 264-6.
E. Bhowmik $(\bowtie) \cdot$ M. Ghosh $\cdot$ T. K. Sabui $\cdot$ R. Mondal Department of Pediatric Medicine, Medical College and Hospital, 88, College Street, Kolkata 700073, India e-mail: eshitabhowmik@gmail.com 